\title{
Spatial and Temporal Incoherence in Scanning Diffraction and Differential Phase Contrast
}

\author{
Mark P. Oxley ${ }^{1,2^{*}}$
}

1. Center for Nanophase Materials Sciences, Oak Ridge National Laboratory, Oak Ridge, TN, USA.

2. Institute for Functional Imaging of Materials, Oak Ridge National Laboratory, Oak Ridge, TN, USA.

* Corresponding author: oxleymp@ornl.gov

Recent developments in segmented detectors and pixelated detectors have led to a resurgence in differential phase contrast (DPC) and scanning diffraction or 4D STEM [1-4]. These new detectors, coupled with aberration correction, allow the use of $\AA$-size probes and promise a wealth of information at atomic resolution. DPC can be used to measure local fields and the large data sets acquired from using pixelated detectors offer possibilities for novel machine learning approaches [5]. However, much of this work overlooks the influence of spatial incoherence (due to a finite electron source size) and temporal incoherence (due to chromatic aberrations and energy variations of the beam). In this work we address these issues for both monolayer and bulk crystalline materials.

Spatial incoherence is routinely added to image simulations using a convolution of some assumed source size distribution, usually Gaussian in form. When constructing realistic simulations based on convergent beam electron diffraction (CBED) patterns, this simple approach is not possible. CBED patterns must be calculated on a suitably fine real-space mesh and then the intensities added together with an appropriate weighting. For this work we will assume a Gaussian distribution with a full-width half-maximum (FWHM) of $S=0.75 \AA$. Temporal incoherence is included by adding CBED patterns over a range of defocus values. The FWHM of the defocus spread is calculated using $\delta f=C_{c} \Delta E / E_{0}$, where $C_{\mathrm{c}}$ is the chromatic aberration coefficient (assumed $1.3 \mathrm{~mm}$ ) and $\Delta E$ is the FWHM of the energy spread (assumed $0.35 \mathrm{eV})$. The size of the defocus spread depends on the incident energy, $E_{0}$.

The magnitude of the deflection of the center of mass (COM) of the CBED patterns is assumed to be related to the local electric field. In Fig. 1 the simulated COM deflection maps are examined for $60 \mathrm{kV}$ incident electrons on monolayer $\mathrm{MoS}_{2}$ using a probe forming aperture of $30 \mathrm{mrad}$. The deflection map for a coherent source is shown in 1(a) and compared to that obtained by adding spatial incoherence 1(b) and temporal incoherence 1(c). The deflection map in 1(d) includes both. There is an obvious reduction in the deflections calculated for all forms of coherence. Spatial coherence results in a larger reduction for the parameters used here. Horizontal scans through the center of the deflection maps are shown in Fig. 1(e). Temporal incoherence seems to preserve the shape of the deflections more than spatial incoherence. An overall reduction in the deflection magnitude of almost a third is observed, suggesting quantitative determination of electric fields from experiment alone may be problematic.

Finally, we consider the use of simulated CBED patterns as input for training sets for deep learning algorithms. A selection of CBED patterns used to construct Fig. 1 are shown in Fig. 2. Spatial incoherence tends to maintain much of the detail of the coherent CBED patterns but reduces the overall contrast. Conversely, temporal incoherence largely maintains the contrast but blurs the features. To construct meaningful training sets for deep learning from simulations, incoherence must be included. Indeed, the degree of incoherence may be another variable if machine parameters are not fully determined.

References: 
[1] N Shibata et al., Nat. Phys. 8 (2012), p. 611.

[2] N Shibata et al., Nat. Commun. 8 (2017), p. 15631.

[3] TJ Pennycook et al., Ultramicroscopy 151 (2015), p. 160.

[4] C Ophus et al., Nat. Commun 7 (2016), p. 10719.

[5] X Li et al., npj Comput. Mater. 5 (2019), p. 5.

[6] Work supported by the U.S. Department of Energy, Office of Basic Energy Sciences, Materials Sciences and Engineering Division.
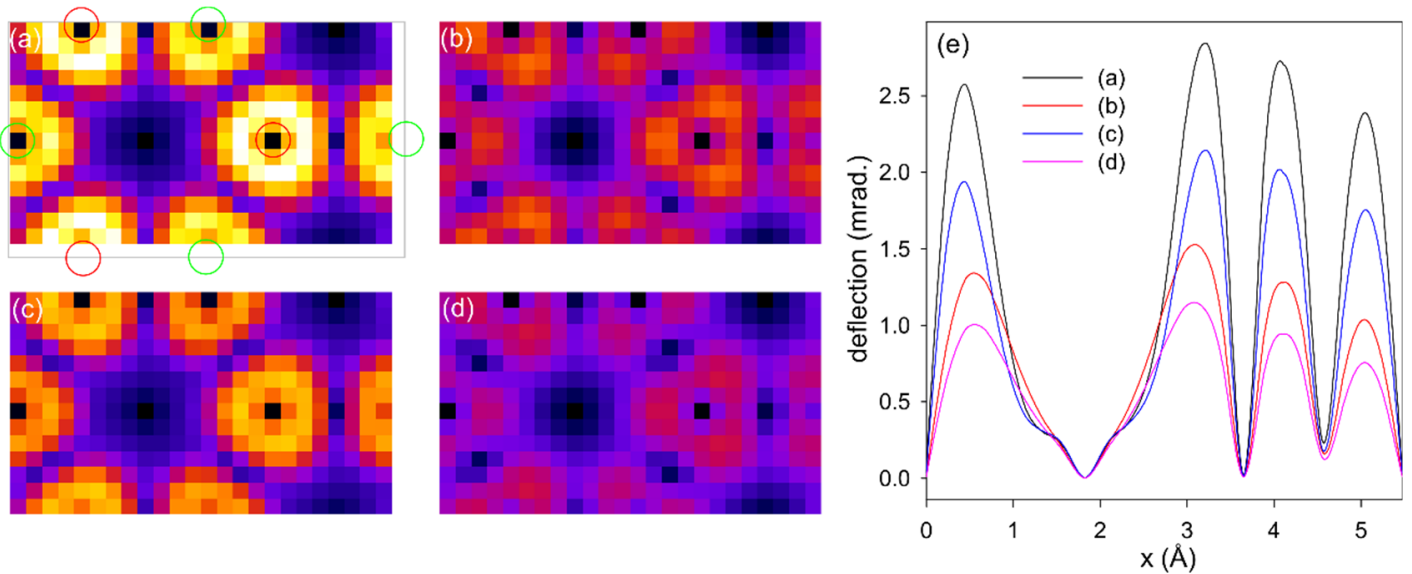

Figure 1. Simulated center of mass deflection maps for $\mathrm{MoS}_{2}$ with $60 \mathrm{kV}$ incident electrons and $30 \mathrm{mrad}$ probe forming aperture. Mo (green circles) and S (red circles) are indicated on (a). (a) fully coherent probe, (b) with spatial incoherence, (c) with temporal incoherence, and (d) with both spatial and temporal incoherence. (e) Horizontal line scans through center of each map.

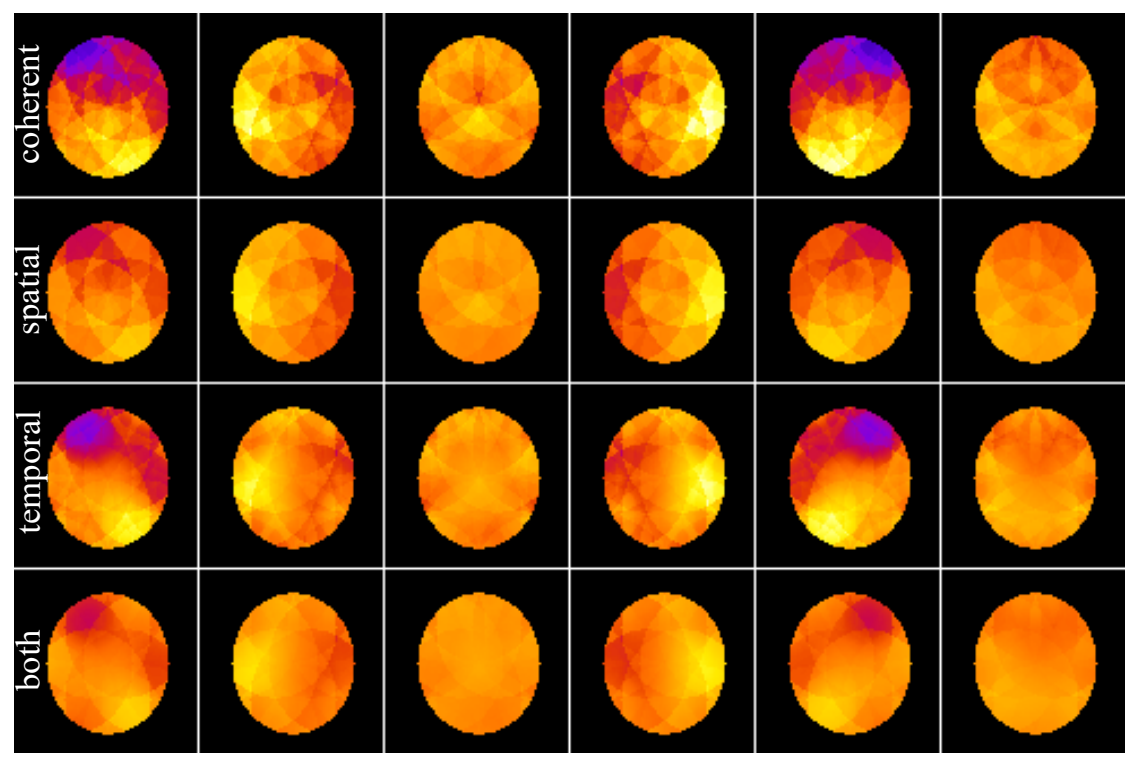

Figure 2. Typical simulated CBED patterns used to construct Fig. 1 with varying degrees of incoherence added. 